This item was submitted to Loughborough's Research Repository by the author.

Items in Figshare are protected by copyright, with all rights reserved, unless otherwise indicated.

\title{
Shared-control for typical driving scenarios
}

PLEASE CITE THE PUBLISHED VERSION

https://doi.org/10.1109/ecc.2016.7810410

PUBLISHER

IEEE @ EUCA

VERSION

AM (Accepted Manuscript)

LICENCE

CC BY-NC-ND 4.0

REPOSITORY RECORD

Jiang, Jingjing, and Alessandro Astolfi. 2019. "Shared-control for Typical Driving Scenarios". figshare. https://hdl.handle.net/2134/36756. 


\title{
Shared-Control for Typical Driving Scenarios
}

\author{
Jingjing Jiang ${ }^{1}$ and Alessandro Astolfi ${ }^{2}$
}

\begin{abstract}
A shared-control algorithm for the kinematic model of a rear-wheel driving car is presented. The design of the shared-controller is based on a hysteresis switch and its properties are established by a Lyapunov-like analysis. The shared-controller guarantees the safety of the car in both predefined, static environments and time-varying environments. The effectiveness of the controller is verified by two studies.
\end{abstract}

\section{INTRODUCTION}

The annual report given by the World Health Organization shows that more than one million people are killed by traffic accidents per year all over the world [1]: this has stimulated researchers and governments to figure out ways to improve the safety of vehicles. Experts claim that the safety of the traditional cars driven completely by the human driver highly depends on the attention, the physical conditions, the experience and the skills of the driver. To reduce the danger caused by these uncertainties and to make driving easier, some scientists and engineers started to study self-driving cars and they have already released some of the achievements [2], [3].

Various methods, such as fuzzy logic controls [4], [5], [6], Model Predictive Controls [7], sliding mode [8], [9], Proportional-Integral-Derivative (PID) controls [10], $H_{\infty}$ controls [11] and machine learning [12], have been used in auto-navigation of cars. In addition, [13] has presented a controller based on two path planning methods and multiple PID controllers to achieve auto-driving, while [14] has utilized fuzzy logic together with PID controllers to navigate a car-like robot. Another robust controller made up of a longitudinal controller and a lateral one to track a given trajectory has been introduced in [15]. The obstacle avoidance problem for vehicles has been studied in [16], where decentralized collision avoidance algorithms based on a switching strategy have been presented. Finally, [17] has proposed a self-driving scheme made up of two steps: finding out the pool of all safe actions and then picking up the most appropriate one on the basis of a cost function.

Even though some driverless cars have already been successfully tested, such as the Google Cars, they still have lots of shortcomings. One of the major limitations is that

\footnotetext{
${ }^{1}$ J. Jiang is with the Department of Electrical and Electronic Engineering, Imperial College London, UK, E-mail: jingjing.jiang10@imperial.ac.uk

${ }^{2} \mathrm{~A}$. Astolfi is with the Dept. of Electrical and Electronic Engineering, Imperial College London, London, SW7 2AZ, UK and the DICII, University of Roma "Tor Vergata", Via del Politecnico 1, 00133 Rome, Italy, E-mail: a.astolfieimperial.ac.uk
}

lots of preparations have to be done before it being driven on a certain road. Data collected from the testing drive has to be studied by computers and humans. Unlike selfdriving cars, this paper studies cars with shared-control that does not keep the human driver out of the control loop. Instead, it combines the human driver and the feedback controller (the auto driver) together. Therefore it is able to take advantages of both controls: the adaptive and interactive task execution ability of the human being and the reliable performance of the feedback controller. The paper [18] has introduced a shared-control law in the "active area" where the vehicle is controlled by the combination of the driver and the local controller. Yet, some researchers have suggested an effective way to help the driver with the human-machine interface: let the driver supervise the process and take critical decisions in high risk situations [19]. Another way to make use of both controllers is to regard the feedback control inputs as suggested control actions as explained in [20]. Furthermore, the paper [21] has presented a shared-control algorithm for the kinematic model of a rear-wheel drive car with knowledge of absolute positions. This paper extends the results given in [21] to cases in which the environment is non-predefined and dynamic.

The paper is organized as follows. Section II describes the model we study in the paper, formulates the shared-control problem, and describes assumptions and definitions used in the rest of the paper. The design of the feedback controller and the sharing function are given in Section III, in which formal properties of the closed-loop system with the sharedcontrol are presented. Section IV gives three examples to illustrate how the shared-control algorithm performs. Finally, some conclusions and suggestions for future work are given in Section V.

\section{PROBLEM STATEMENT}

In this section we formulate the shared-control problem for the kinematic model of a rear-wheel driving car, the dynamics of which can be described by the equations

$$
\begin{aligned}
& \dot{x}=v_{s} \cos \theta, \\
& \dot{y}=v_{s} \sin \theta, \\
& \dot{\theta}=\frac{v_{s} \tan \phi}{l}, \\
& \dot{\phi}=\omega_{s},
\end{aligned}
$$

where $(x, y)$ denotes the Cartesian coordinates of the middle of the rear-axle, $\theta$ is the heading angle of the car, $\phi$ represents the angle between the front-wheel and the heading direction 


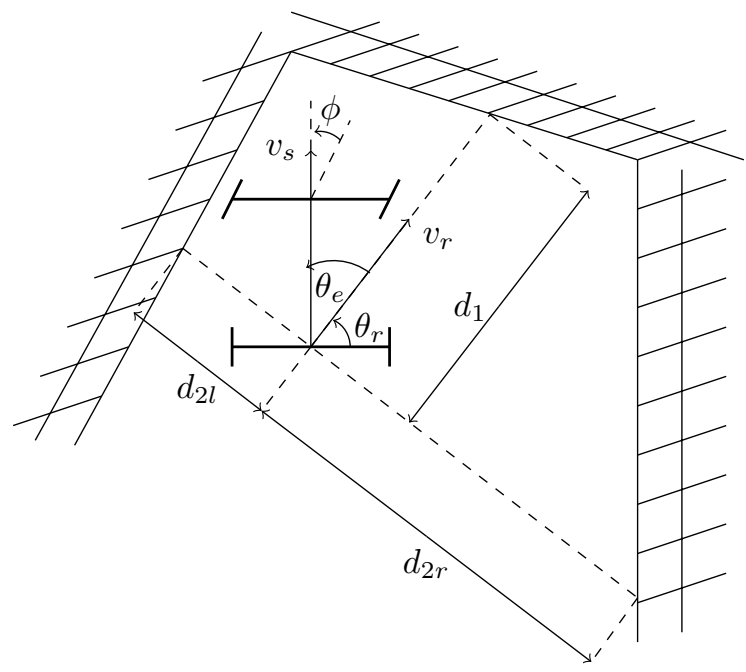

Fig. 1. Definitions of $d_{1}, d_{2 l}, d_{2 r}, \theta_{e}$ and $\phi$ (shadowed region: unfeasible region, $v_{r}$ : reference forward velocity, $\theta_{r}$ : reference forward angle, $v_{s}$ : actual forward velocity). Note that $\theta=\theta_{e}+\theta_{r}$.

of the car, $l$ is the distance between the middle of the rearaxle and that of the front-axle, $v_{s}$ and $\omega_{s}$ are the sharedcontrol inputs of the system, describing the linear velocity of the car and the angular velocity of the front-wheels, respectively.

As discussed in Section I this paper is an extension of [21] in that the shared-control problem is solved for nonpredefined dynamic environments. In addition, different from [21], we assume that no absolute position is measured. Instead, we measure the distance to obstacles along (i.e. $d_{1}$ ) and orthogonal to (i.e. $d_{2}=\min \left\{d_{2 l}, d_{2 r}\right\}$ ) the direction of the reference forward velocity, the distance to the obstacle along the current forward direction (i.e. D), the difference between the actual and the reference heading angle (i.e. $\left.\theta_{e}=\theta-\theta_{r}\right)$ and the steering angle $\phi$, see Fig. 1. Note that $\theta_{r}$ is measured relative to the positive $x$-direction.

Define the shared-control input as a function of $t, D, d_{1}, d_{2}, \theta_{e}$ and $\phi$ as

$$
\begin{array}{r}
v_{s}\left(D, d_{1}, d_{2}, \theta_{e}, \phi, t\right)=(1-k) v_{f}\left(d_{1}, d_{2}, \theta_{e}, \phi, t\right) \\
+k v_{h}(\phi, t), \\
\omega_{s}\left(D, d_{1}, d_{2}, \theta_{e}, \phi, t\right)=(1-k) \omega_{f}\left(d_{1}, d_{2}, \theta_{e}, \phi, t\right) \\
+k \omega_{h}(t),
\end{array}
$$

where $u_{h}=\left[v_{h}, \omega_{h}\right]^{T}$, denoted as $h$-control, describes the human action, $u_{f}=\left[v_{f}, \omega_{f}\right]^{T}$, denoted as $f$-control, represents the feedback-control action and $k$, denoted as sharing function, quantifies how the control authority is shared between the operator and the feedback controller. Similarly to [21] we use the name s-closed-loop to denote the closed-loop system described by (1) with the shared-control input defined in (2) and the name $h$-closed-loop to denote the closed-loop system controlled by the human operator alone, i.e.

$$
\begin{aligned}
& \dot{x}=v_{h} \cos \theta, \\
& \dot{y}=v_{h} \sin \theta, \\
& \dot{\theta}=\frac{v_{h} \tan \phi}{l}, \\
& \dot{\phi}=\omega_{h},
\end{aligned}
$$

Suppose $\mathcal{P}_{a}(t) \subset \mathbb{R}^{2}$ is a dynamic, closed and compact set describing the "admissible Cartesian configurations" for the system (1) and $u_{h}$ is a given h-control. The shared-control problem for the kinematic model of a rear-wheel driving car can be formulated as follows.

Given the system (1), an admissible configuration set $\mathcal{P}_{a}(t)$ and an h-control, find (if possible)

- an f-control $u_{f}$;

- a sharing function $k$;

- a safe set $\mathcal{R}_{s}\left(v_{h}, t\right) \triangleq \mathcal{P}_{a}(t) \times \mathcal{H}_{s} \times \mathcal{A}_{s} \subset \mathcal{P}_{a}(t) \times \mathcal{H} \times$ $\mathcal{A} \triangleq \mathcal{R}\left(v_{h}, t\right)$

where $\mathcal{H}\left(\mathcal{H}_{s}\right)$ and $\mathcal{A}\left(\mathcal{A}_{s}\right)$ are the set of heading angles (safe heading anlges) and wheeling angles (safe wheeling angles) respectively, such that the s-closed-loop system (1)-(2) has the following properties.

P1) The set $\mathcal{R}\left(v_{h}, t\right)$ is forward invariant.

P2) Let $\Omega_{s}$ and $\Omega_{h}$ be the $\Omega$-limit set of the s-closed-loop and h-closed-loop, respectively. Then

$$
\Omega_{s}= \begin{cases}\Omega_{h} & \text { if } \Omega_{h} \subset \mathcal{R}_{s}\left(v_{h}, t\right), \\ \Pi_{\mathcal{R}_{s}}\left(\Omega_{h}\right) & \text { if } \Omega_{h} \not \subset \mathcal{R}_{s}\left(v_{h}, t\right),\end{cases}
$$

where $\Pi_{\mathcal{R}_{s}}\left(\Omega_{h}\right)$ is the projection of $\Omega_{h}$ into the set $\mathcal{R}_{s}\left(v_{h}, t\right)$, which will be defined in Section III-A.

P3) $u_{s}=u_{h}$ if the state of the system belongs to $\mathcal{R}_{s}\left(v_{h}, t\right)$.

Note that for any fixed $D$ and $v_{h}$, the sets $\mathcal{H}_{s}$ and $\mathcal{A}_{s}$ are the sets of all possible heading angles and front-wheel angles such that the car cannot hit the boundary of $\mathcal{P}_{a}$ within a short period of time.

Assumption 1: The projection of the car in the $(x, y)$-plane is a rectangle with length $l$ and width $w$, where $l$ denotes the distance between the middle of the front-axle and that of the rear-axle and $w$ denotes the distances between the centers of two rear wheels (or the centers of two front wheels).

Assumption 2: The human-generated function $d_{d}(t)=$ $\left[d_{d 1}(t) ; d_{d 2}(t)\right]^{T}$, representing the desired distances to the relative obstacles (i.e. the obstacles along and orthogonal to the the reference forward direction), is sufficiently smooth.

Definition 1: Suppose the time-varying set $\mathcal{P}(t)$ is closed for any $t \geq 0$. Then it is continuous with respect to time if

$$
\lim _{t \rightarrow t_{0}}\left[\max _{x \in \mathcal{P}(t)} d\left(x, \mathcal{P}\left(t_{0}\right)\right)\right]=0,
$$


where $d\left(x, \mathcal{P}\left(t_{0}\right)\right)$ represents the distance between the point $x$ and the set $\mathcal{P}\left(t_{0}\right)$.

Assumption 3: The admissible Cartesian configuration set is always non-empty, i.e. $\mathcal{P}_{a}(t) \neq \emptyset \quad \forall t \geq 0$. In addition, it is continuous with respect to time.

Definition 2: The function $f(y(t), x(t), t)=$ $\operatorname{atg}(y(t), x(t), t)$ is a continuous function defined as

$$
\operatorname{atg}(y(t), x(t), t)=\operatorname{atan}(y(t), x(t))+2 \alpha(t) \pi,
$$

where atan is the four quadrant arctan function, $\alpha(0)=0$ and

$$
\alpha(t)= \begin{cases}\alpha(t-\delta t)+1, & \text { if } a=-2 \pi \\ \alpha(t-\delta t)-1, & \text { if } a=2 \pi \\ \alpha(t-\delta t), & \text { else }\end{cases}
$$

with $a=\lim _{\delta t \rightarrow 0^{+}} \operatorname{atan}(y(t), x(t))-\operatorname{atan}(y(t-\delta t), x(t-\delta t))$ for all $t>0$.

Note that the definition of the function $\operatorname{atg}(y(t), x(t), t)$ is close to that of the standard four quadrant arctan function $\operatorname{atan}(y(t), x(t))$ except that $\operatorname{atg}(y(t), x(t), t)$ is a continuous function with range equal to $(-\infty, \infty)$.

\section{DESIGN OF THE SHARED-CONTROLLER}

In this section we give a solution to the shared-control problem stated in Section II. This relies on two steps: the design of the feedback controller, which is given in Section III-A, and the definition of how the control authority is shared between the driver and the feedback controller, which is presented in Section III-B.

\section{A. Design of the Feedback Controller}

According to the definition, $d_{2}=d_{2 l}$ or $d_{2}=d_{2 r}$. If $d_{2}=$ $d_{2 l}$, then the dynamics of the closed-loop system controlled by the feedback controller can be described by the equations

$$
\begin{aligned}
\dot{d}_{1} & =-v_{f} \cos \theta_{e} \\
\dot{d}_{2} & =-v_{f} \sin \theta_{e} \\
\dot{\theta}_{e} & =\frac{v_{f} \tan \phi-v_{r} \tan \phi_{r}}{l} \\
\dot{\phi} & =\omega_{f} .
\end{aligned}
$$

On the other hand, if $d_{2}=d_{2 r}$, then the dynamics of the closed-loop system with feedback control can be described by the equations

$$
\begin{aligned}
\dot{d}_{1} & =-v_{f} \cos \theta_{e}, \\
\dot{d}_{2} & =v_{f} \sin \theta_{e}, \\
\dot{\theta}_{e} & =\frac{v_{f} \tan \phi-v_{r} \tan \phi_{r}}{l}, \\
\dot{\phi} & =\omega_{f} .
\end{aligned}
$$

In the case in which $d_{2 l}=d_{2 r}$, we define $d_{2}$ as $d_{2}=$ $d_{2 l}$. Without loss of generality, we only study the case in which $d_{2}=d_{2 l}$. By Assumption 1 the position constraints $(x(t), y(t)) \in \mathcal{P}_{a}(t)$ can be rewritten as

$$
d_{i}(t) \geq \tilde{d}_{i}
$$

where $\tilde{d}_{i}$ is a positive constant for all $i \in\{1,2\}$. One choice for $\tilde{d}_{1}$ and $\tilde{d}_{2}$ is $\tilde{d}_{1}=\tilde{d}_{2}=\sqrt{l^{2}+\left(\frac{w}{2}\right)^{2}}$.

Define the variable $z=\left[z_{1}, z_{2}\right]^{T}$ as

$$
z_{i}=\log \frac{d_{i}-\tilde{d}_{i}}{d_{r_{i}}-\tilde{d}_{i}}, \forall i \in\{1,2\},
$$

where $d_{r_{1}}$ and $d_{r_{2}}$ describe the reference trajectories for the state $d_{1}$ and $d_{2}$, respectively, and are defined as

$$
d_{r_{i}}= \begin{cases}d_{d_{i}}, & \text { if } d_{d_{i}} \geq\left(1-\frac{\sqrt{2}}{2}\right) r+\epsilon+\tilde{d}_{i} \\ \epsilon+\tilde{d}_{i}, & \text { if } d_{d_{j}} \leq(1-\sqrt{2}) r+\epsilon+\tilde{d}_{i} \\ m_{i}, & \text { otherwise }\end{cases}
$$

for all $i \in\{1,2\}$, with $m_{i}=r+\epsilon+\tilde{d}_{i}-$ $\sqrt{r^{2}-\left[(\sqrt{2}-1) r-\epsilon+d_{d_{i}}-\tilde{d}_{i}\right]^{2}}$ and a sufficiently small positive constant $\epsilon$. In the trajectory-tracking cases, the feedback controller and the human driver share the same information about reference signals, i.e. $d_{d}=\left[d_{d_{1}}, d_{d_{2}}\right]^{T}$ is known to the feedback controller. On the other hand, in the free-driving cases, $d_{d}(t)$ is calculated from $u_{h}(t)$. From the definition of $d_{r}$ given by (6) it is obvious that $d_{r_{i}}$ is a smooth function with all values larger than $\tilde{d}_{i}$ for $i \in\{1,2\}$. Therefore, $\dot{d}_{r_{1}}$ exists and

$$
v_{r}=-\dot{d}_{r_{1}}
$$

where $v_{r}$ is the reference forward velocity.

Since absolute positions are not available, we define the safe subset $\mathcal{R}_{s}$ in the $\left(d_{1}, d_{2}, \theta_{e}, \phi\right)$ coordinates. Consider any point $\left(d_{d_{1}}, d_{d_{2}}, \theta_{d}, \phi_{d}\right)$ in the $\Omega$-limit set of the h-closedloop system, i.e. $\left(d_{d_{1}}, d_{d_{2}}, \theta_{d}, \phi_{d}\right) \in \Omega_{h}$, then the projection of it into the safe subset $\mathcal{R}_{s}$ is defined as

$$
\Pi_{\mathcal{R}_{s}}\left(d_{d_{1}}, d_{d_{2}}, \theta_{d}, \phi_{d}\right)=\left(d_{r_{1}}, d_{r_{2}}, \theta_{d}, \phi_{d}\right),
$$

where $d_{r_{1}}$ and $d_{r_{2}}$ are defined by (6). Hence, the projection of $\Omega_{h}$ into the set $\mathcal{R}_{s}$ is defined by

$$
\Pi_{\mathcal{R}_{s}}\left(\Omega_{h}\right)=\left\{\begin{array}{l|l}
s \in \mathcal{R}_{s} & s=\Pi_{\mathcal{R}_{s}}\left(d_{d_{1}}, d_{d_{2}}, \theta_{d}, \phi_{d}\right), \\
\forall\left(d_{d_{1}}, d_{d_{2}}, \theta_{d}, \phi_{d}\right) \in \Omega_{h}
\end{array}\right\} .
$$

Using the variable $z$ system (3) can be written as

$$
\begin{aligned}
\dot{z}_{1} & =\frac{v_{r}}{d_{r_{1}}-\tilde{d}_{1}}-\frac{v_{f} \cos \theta_{e}}{d_{1}-\tilde{d}_{1}}, \\
\dot{z}_{2} & =-\frac{v_{f} \sin \theta_{e}}{d_{2}-\tilde{d}_{2}}, \\
\dot{\theta}_{e} & =\frac{v_{f} \tan \phi-v_{r} \tan \phi_{r}}{l}, \\
\dot{\phi} & =\omega_{f} .
\end{aligned}
$$


Let

$$
\begin{aligned}
\theta_{e}^{*} & =\operatorname{atg}\left(\gamma_{2}\left(d_{2}-\tilde{d}_{2}\right) z_{2},\left(d_{1}-\tilde{d}_{1}\right)\left(\frac{v_{r}}{d_{r_{1}}-\tilde{d}_{1}}+\gamma_{1} z_{1}\right)\right), \\
\alpha & =\sqrt{\left(\gamma_{2}\left(d_{2}-\tilde{d}_{2}\right) z_{2}\right)^{2}+\left[\left(d_{1}-\tilde{d}_{1}\right)\left(\frac{v_{r}}{d_{r_{1}}-\tilde{d}_{1}}+\gamma_{1} z_{1}\right)\right]^{2}}, \\
\phi^{*} & =\operatorname{atg}\left(\begin{array}{c}
\frac{l z_{2}}{d_{2}-\tilde{d}_{2}} \cos \frac{\theta_{e}+\theta_{e}^{*}}{2} \operatorname{sinc} \frac{\theta_{e}-\theta_{e}^{*}}{2} \\
-\frac{l z_{1}}{d_{1}-\tilde{d}_{1}} \sin \frac{\theta_{e}+\theta_{e}^{*}}{2} \operatorname{sinc} \frac{\theta_{e}-\theta_{e}^{*}}{2}, 1 \\
+\frac{v_{r} \tan \phi_{r}}{\alpha}+\frac{l \dot{\theta}_{e}^{*}}{\alpha}
\end{array}\right),
\end{aligned}
$$

where $\gamma_{1}>0, \gamma_{2}>0$.

Consider the Lyapunov function candidate

$$
L_{l}\left(z_{1}, z_{2}, \theta_{e}, \phi, t\right)=\frac{1}{2}\left\{\begin{array}{c}
z_{1}^{2}+z_{2}^{2}+\left(\theta_{e}-\theta_{e}^{*}\right)^{2} \\
+\left(\tan \phi-\tan \phi^{*}\right)^{2}
\end{array}\right\},
$$

and choose $v_{f}$ and $\omega_{f}$ such that $\dot{L}(t) \leq 0$ for all $t \geq 0$ and $\dot{L} \equiv 0$ indicates $\left(z_{1}, z_{2}, \theta_{e}, \phi\right)=\left(0,0, \theta_{e}^{*}, \phi^{*}\right)$. One such a choice is given by

$$
\begin{aligned}
& v_{f}=\alpha, \\
& \omega_{f}=\cos ^{2} \phi\left[\begin{array}{c}
\frac{\dot{\phi}^{*}}{\cos ^{2} \phi^{*}}-\frac{v\left(\theta_{e}-\theta_{e}^{*}\right)}{l} \\
-\gamma_{3}\left(\tan \phi-\tan \phi^{*}\right)
\end{array}\right],
\end{aligned}
$$

where $\gamma_{3}>0$, yielding

$$
\dot{L}_{l}^{i}=-\gamma_{1} z_{1}^{2}-\gamma_{2} z_{2}^{2}-\gamma_{3}\left(\tan \phi-\tan \phi^{*}\right)^{2} \leq 0 .
$$

This can be transformed into the $\left(d_{1}, d_{2}, \theta_{e}, \phi\right)$ coordinates by

$$
\begin{aligned}
& v_{f}=\sqrt{\begin{array}{c}
\left(d_{1}-\tilde{d}_{1}\right)^{2}\left(\frac{v_{r}}{d_{r_{1}}-\tilde{d}_{1}}+\gamma_{1} \log \frac{d_{1}-\tilde{d}_{1}}{d_{r_{1}}-\tilde{d}_{1}}\right)^{2} \\
+\gamma_{2}^{2}\left(d_{2}-\tilde{d}_{2}\right)^{2} \log ^{2} \frac{d_{2}-\tilde{d}_{2}}{d_{r_{2}}-\tilde{d}_{2}}
\end{array}}, \\
& \omega_{f}=\cos ^{2} \phi\left[\begin{array}{c}
\frac{\dot{\phi}^{*}}{\cos ^{2} \phi^{*}}-\frac{v\left(\theta_{e}-\theta_{e}^{*}\right)}{l} \\
-\gamma_{3}\left(\tan \phi-\tan \phi^{*}\right)
\end{array}\right],
\end{aligned}
$$

where

$$
\begin{gathered}
\theta_{e}^{*}=\operatorname{atg}\left(\begin{array}{c}
\gamma_{2}\left(d_{2}-\tilde{d}_{2}\right) \log \frac{d_{2}-\tilde{d}_{2}}{d_{r_{2}}-\tilde{d}_{2}}, \\
\left(d_{1}-\tilde{d}_{1}\right)\left(\frac{v_{r}}{d_{r_{1}}-\tilde{d}_{1}}+\gamma_{1} \log \frac{d_{1}-\tilde{d}_{1}}{d_{r_{1}}-\tilde{d}_{1}}\right)
\end{array}\right), \\
\phi^{*}=\operatorname{atg}\left(\begin{array}{c}
\frac{l}{d_{2}-\tilde{d}_{2}} \log \frac{d_{2}-\tilde{d}_{2}}{d_{r_{2}}-\tilde{d}_{2}} \cos \frac{\theta_{e}+\theta_{e}^{*}}{2} \operatorname{sinc} \frac{\theta_{e}-\theta_{e}^{*}}{2} \\
-\frac{l}{d_{1}-\tilde{d}_{1}} \log \frac{d_{1}-\tilde{d}_{1}}{d_{r_{1}}-\tilde{d}_{1}} \sin \frac{\theta_{e}+\theta_{e}^{*}}{2} \operatorname{sinc} \frac{\theta_{e}-\theta_{e}^{*}}{2} \\
+\frac{v_{r} \tan \phi_{r}}{v_{f}}+\frac{l \dot{\theta}_{e}^{*}}{v_{f}}
\end{array}\right.
\end{gathered}
$$

and $d_{r}=\left[d_{r_{1}}, d_{r_{2}}\right]^{T}$ is given by (6).
Note that $v_{f}$ is always non-negative since we do not consider the 'reverse gear' of the car in the paper.

Lemma 1: Consider the closed-loop system (3) controlled by the feedback controller (9) with $d_{r}$ and $v_{r}$ given by (6) and (7), respectively. Assume the initial position is feasible, i.e. $(x(0), y(0)) \in \mathcal{P}_{a}, d_{1}(0)>\tilde{d}_{1}$ and $d_{2}(0)>\tilde{d}_{2}$. Then the closed-loop system has the following properties.

- $d_{1}(t)>\tilde{d}_{1}, d_{2}(t)>\tilde{d}_{2}$ for all $t \geq 0$

- $\lim _{t \rightarrow \infty}\left(d_{1}(t)-d_{r_{1}}(t)\right)=\lim _{t \rightarrow \infty}\left(d_{2}(t)-d_{r_{2}}(t)\right)=0$;

- $|\phi(t)|<\frac{\pi}{2}$ for all $t \geq 0$.

\section{B. Shared Control Theorem}

For any given human input $v_{h}$, the safe, hysteresis and dangerous subsets, $\mathcal{R}_{s}, \mathcal{R}_{h}$ and $\mathcal{R}_{d}$, are defined by equations (10) given at the top of next page, where $D$ is the distance to the obstacle along the current forward direction and $b_{2}>$ $b_{1}>0$ are constants selected by the user.

The sharing function $k$ can then be defined as

$$
k= \begin{cases}1, & \left(d_{1}, d_{2}, \theta_{e}, \phi\right) \in \mathcal{R}_{s}\left(v_{h}\right) \\ l, & \left(d_{1}, d_{2}, \theta_{e}, \phi\right) \in \mathcal{R}_{h}\left(v_{h}\right) \\ 0, & \left(d_{1}, d_{2}, \theta_{e}, \phi\right) \in \mathcal{R}_{d}\left(v_{h}\right)\end{cases}
$$

where

$l= \begin{cases}1, & \text { if }\left(d_{1}, d_{2}, \theta_{e}, \phi\right) \text { enters } \mathcal{R}_{h}\left(v_{h}\right) \text { from } \mathcal{R}_{s}\left(v_{h}\right), \\ 0, & \text { if }\left(d_{1}, d_{2}, \theta_{e}, \phi\right) \text { enters } \mathcal{R}_{h}\left(v_{h}\right) \text { from } \mathcal{R}_{d}\left(v_{h}\right)\end{cases}$

Finally, the overall shared-control input $u_{s}=\left[v_{s}, \omega_{s}\right]^{T}$ is given by

$$
u_{s}=(1-k) u_{f}\left(d, d_{r}, \theta_{e}, \phi, v_{r}\right)+k u_{h} .
$$

Theorem 1: Consider the kinematic model (1) of a rearwheel driving car with the shared-control input given by (9)-(11)-(12). Assume the initial condition is feasible, i.e. $(x(0), y(0)) \in \mathcal{P}_{a}(0), d_{1}(0)>\tilde{d}_{1}$ and $d_{2}(0)>\tilde{d}_{2}$, and $u_{h}$ is a given $\mathrm{h}$-control. Then there exists $\gamma_{i}>0$ for all $i \in\{1,2,3\}$ such that the closed-loop system has the following properties.

1) $d_{1}(t)>\tilde{d}_{1}$ and $d_{2}(t)>\tilde{d}_{2}$ for all $t \geq 0$;

2) $\Omega_{s}=\Pi_{\mathcal{R}_{s}}\left(\Omega_{h}\right)$;

3) $u_{s}(t)=u_{h}(t)$ for all $t$ such that $\left(d_{1}(t), d_{2}(t), \theta_{e}(t), \phi(t)\right) \in \mathcal{R}_{s}$.

Remark 1: If $\tilde{d}_{1}=\tilde{d}_{2}=\sqrt{l^{2}+\left(\frac{w}{2}\right)^{2}}$, the car is unable to stop next to the boundary of the admissible Cartesian configuration set. However, this issue can easily be solved by changing the values of $\tilde{d}_{1}$ and $\tilde{d}_{2}$ to $l$ and $\frac{w}{2}$, respectively, when $\theta_{e}$ is close to 0 .

Remark 2: If the car is far away from the boundary of its admissible configuration set, the system state belongs to the 


$$
\begin{gathered}
\mathcal{R}_{s}\left(v_{h}\right)=\left\{\left(d_{1}, d_{2}, \theta_{e}, \phi\right) \in \mathbb{R}^{+} \times \mathbb{R}^{+} \times \mathbb{S} \times \mathbb{S}: v_{h} \leq \frac{1}{b_{2}-D}-\frac{1}{b_{2}} \text { if } D \leq b_{2}\right\} \\
\mathcal{R}_{h}\left(v_{h}\right)=\left\{\begin{array}{r}
\left(d_{1}, d_{2}, \theta_{e}, \phi\right) \in \mathbb{R}^{+} \times \mathbb{R}^{+} \times \mathbb{S} \times \mathbb{S}: \quad \frac{1}{b_{2}-D}-\frac{1}{b_{2}}<v_{h}<\frac{1}{b_{1}-D}-\frac{1}{b_{1}} \text { and } D \leq b_{1} \\
\text { or } v_{h}>\frac{1}{b_{2}-D}-\frac{1}{b_{2}} \text { and } b_{1} \leq D \leq b_{2}
\end{array}\right\} \\
\mathcal{R}_{d}\left(v_{h}\right)=\left\{\left(d_{1}, d_{2}, \theta_{e}, \phi\right) \in \mathbb{R}^{+} \times \mathbb{R}^{+} \times \mathbb{S} \times \mathbb{S}: v_{h} \geq \frac{1}{b_{1}-D}-\frac{1}{b_{1}} \text { and } 0 \leq D \leq b_{1}\right\}
\end{gathered}
$$

safe subset $\mathcal{R}_{s}$ and the control authority is held completely by the driver. In other words, we only need to design the feedback controller if there exists one $i \in\{1,2\}$ such that $d_{i} \leq B$, where $B$ is a positive constant. Therefore, we can modify $d_{i}$ to $\hat{d}_{i}=\min \left(d_{i}, B\right)$.

\section{CASE STUDIES}

This section discusses two case studies: overtaking and turning. We assume that the projection of the car in $(x, y)$-plane is a rectangle with size $1 m \times 1.5 \mathrm{~m}$. These cases are simulated using MATLAB SIMULINK and the results demonstrate that the safety of the car with the shared-controller is guaranteed even if the driver behaves dangerously.

\section{A. Overtaking}

Consider the kinematic model of the rear-wheel driving car described by the equations (1) traveling on a dual-lane oneway carriageway. Assume the driver sitting inside the car with the shared-controller is overtaking the car in front of him, but does not turn his/her wheel properly.

Simulation results are shown in Fig. 2, from which we see that the feedback controller is active when the controlled car is close to the car in front of it with a high incorrect heading speed. Note that the shared-controller passes the control authority back to the driver again when the car is running on the overtaking lane (the right-hand lane). After successfully overtaking the blue car, the driver drives the car back to the left lane and continues his journey.

\section{B. Turning Into a Fast Road}

Consider the same model as in Section IV-A with the sharedcontrol given by (9)-(11)-(12). The car is approaching a junction with a main road. The driver aims to turn left and to continue the journey on the main road. However, he/she does not check carefully the running traffic.

Simulation results are illustrated in Fig. 3, from which we see that the feedback-controller is active when the car approaches the junction and the sensors detect that there is not enough space for the car to make a turn and join the traffic on the
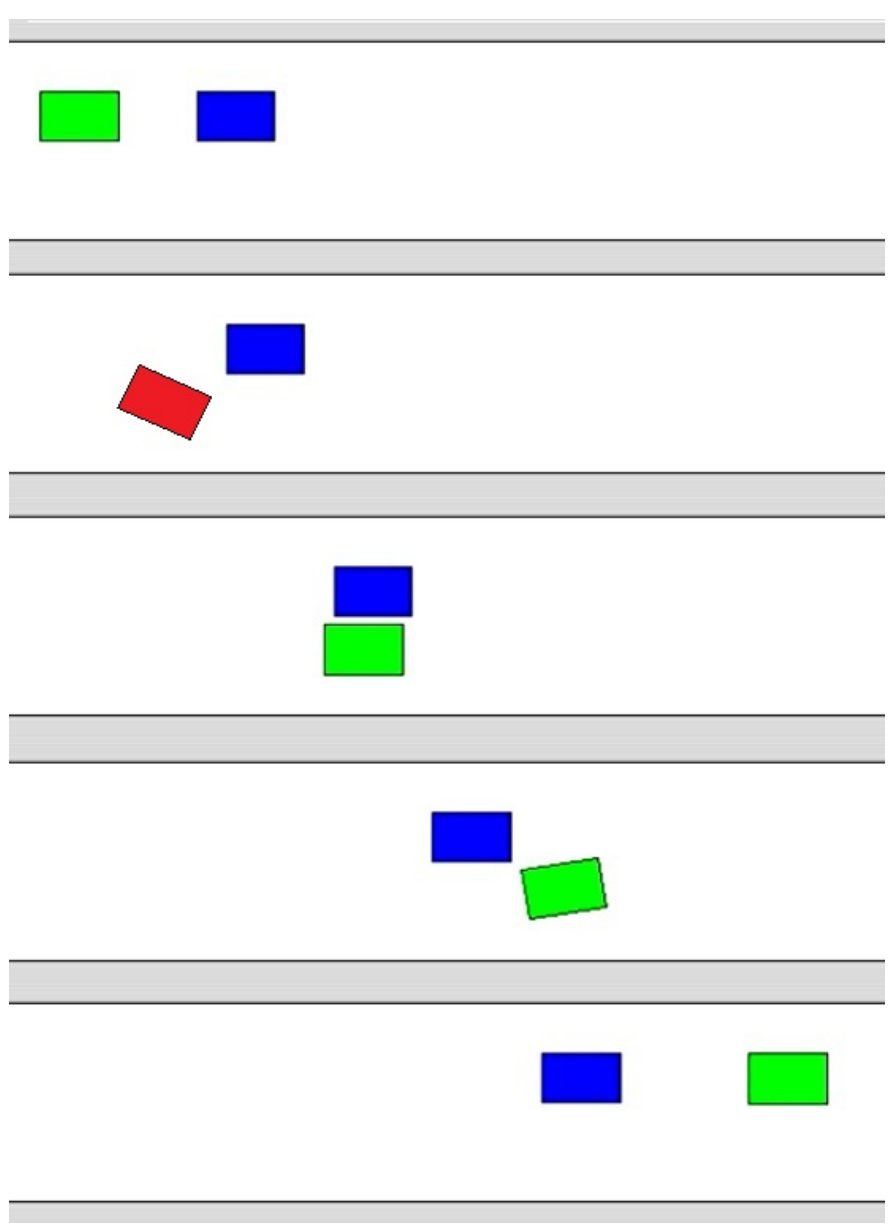

Fig. 2. Snapshots of the car path with the shared-control in the $(x, y)$ plane for the set $\mathcal{P}_{a}$ represented by the white area. Green car: the controlled car (the feedback controller is not active). Red car: the controlled car (the feedback controller is active). Blue car: the other vehicle on the road.

main road. Therefore, with the help of the shared-control, the car gently stops at the junction and waits for a proper space to turn.

\section{CONCLUSIONS}

We have developed a solution to the shared-control problem for the kinematic model of a rear-wheel driving car, without knowledge of absolute positions, for which the set of the feasible Cartesian positions is dynamic and not predefined. 

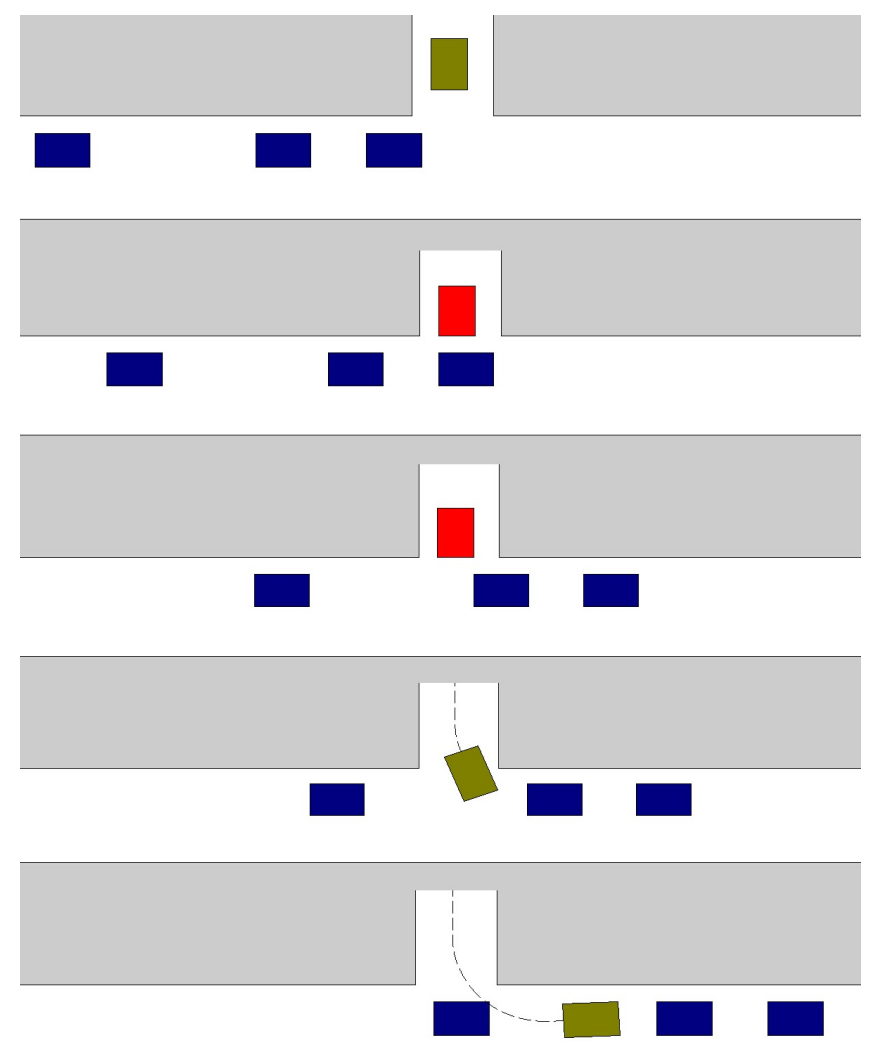

Fig. 3. Snapshots of the car path with the shared-control in the $(x, y)$ plane for the set $\mathcal{P}_{a}$ represented by the white area. Green car: the controlled car (the feedback controller is not active). Red car: the controlled car (the feedback controller is active). Blue cars: the other vehicles on the road. Dashed line: $(x, y)$-trajectory of the controlled car.

The algorithm integrates the human inputs and the feedback control inputs based on a hysteresis switch. Two simple case studies shown in Section IV demonstrate the effectiveness of the shared-control law. Future work will focus on systems with model uncertainties, such as bounded disturbances in the system dynamics.

\section{REFERENCES}

[1] WHO, Global Status Report on Road Safety 2013: Supporting a Decade of Action. Luxemourg.

[2] E. Guizzo, "How googles self-driving car works," IEEE Spectrum Online, October, vol. 18, 2011.

[3] K. Jo, J. Kim, D. Kim, C. Jang, and M. Sunwoo, "Development of autonomous carpart ii: A case study on the implementation of an autonomous driving system based on distributed architecture," IEEE Trans. on Industrial Electronics, vol. 62, no. 8, pp. 5119-5132, 2015.

[4] I. Baturone, F. Moreno-Velo, S. Sanchez-Solano, and A. Ollero, "Automatic design of fuzzy controllers for car-like autonomous robots," IEEE Trans. on Fuzzy Systems, vol. 12, no. 4, pp. 447-465, 2004

[5] T. Li and S. Chang, "Autonomous fuzzy parking control of a car-like mobile robot," IEEE Trans. on Systems, Man and Cybernetics, Part A: Systems and Humans, vol. 33, no. 4, pp. 451-465, 2003.
[6] D. Ho and J. Garibaldi, "A novel fuzzy inferencing methodology for simulated car racing," in Proc. of IEEE International Conference on Fuzzy Systems, 2008, pp. 1907-1914.

[7] R. Kianfar, B. Augusto, A. Ebadighajari, U. Hakeem, J. Nilsson, A. Raza, R. Tabar, N. Irukulapati, C. Englund, P. Falcone, S. Papanastasiou, L. Svensson, and H. Wymeersch, "Design and experimental validation of a cooperative driving system in the grand cooperative driving challenge," IEEE Trans. on Intelligent Transportation Systems, vol. 13, no. 3, pp. 994-1007, 2012.

[8] N. Ghani and Y. Sam, "Sliding mode control of active car steering with various boundary layer thickness and disturbances," in Proc. of IEEE Conference on Industrial Electronics and Applications, 2007, pp. 2494-2499.

[9] N. Yagiz, Y. Hacioglu, and Y. Taskin, "Fuzzy sliding-mode control of active suspensions," IEEE Trans. on Industrial Electronics, vol. 55, no. 11, pp. 3883-3890, 2008.

[10] J. Tjonnas and T. Johansen, "Stabilization of automotive vehicles using active steering and adaptive brake control allocation," IEEE Trans. on Control Systems Technology, vol. 18, no. 3, pp. 545-558, 2009.

[11] C. Hwang and L. Chang, "Trajectory tracking and obstacle avoidance of car-like mobile robots in an intelligent space using mixed $\mathrm{h} 2 / \mathrm{h}$ decentralized control," IEEE Trans. on Mechatronics, vol. 12, no. 3 , pp. 345-352, 2007.

[12] L. Ellis, N. Pugeault, K. Ofjall, J. Hedborg, R. Bowden, and M. Felsberg, "Autonomous navigation and sign detector learning," in Proc. of IEEE Workshop on Robot Vision, 2013, pp. 144-151.

[13] S. Thrun, "Toward robotic cars," Communications of the ACM, vol. 53, no. 4, pp. 99-106, 2010.

[14] H. Abatari and A. Tafti, "Using a Fuzzy PID Controller for the Path Following of a Car-Like Mobile Robot," in Proc. of RSI/ISM International Conference on Robotics and Mechatronics, 2013, pp. 189-193.

[15] C. Massera Filho, D. Wolf, V. Grassi, and F. Osorio, "Longitudinal and lateral control for autonomous ground vehicles," in Proc. of IEEE Symposium on Intelligent Vehicles, 2014, pp. 588-593.

[16] C. Hwang, L. Chang, and Y. Yu, "Network-based fuzzy decentralized sliding-mode control for car-like mobile robots," IEEE Trans. on Industrial Electronics, vol. 54, no. 1, pp. 574-585, 2007.

[17] A. Furda and L. Vlacic, "Towards increased road safety: Realtime decision making for driverless city vehicles," in Proc. of IEEE Conference on Systems, Man and Cybernetics, 2009, pp. 2421-2426.

[18] J. Alonso-Mora, P. Gohl, S. Watson, R. Siegwart, and P. Beardsley, "Shared control of autonomous vehicles based on velocity space optimization," in Proc. of IEEE Conference on Robotics and Automation, 2014, pp. 1639-1645.

[19] A. Franchi, C. Secchi, M. Ryll, H. Bulthoff, and P. Giordano, "Shared Control : Balancing Autonomy and Human Assistance with a Group of Quadrotor UAVs," IEEE Robotics and Automation Magazine, vol. 19, no. 3, pp. 57-68, 2012.

[20] M. Michalek and M. Kielczewski, "Helping a driver in backward docking with n-trailer vehicles by the passive control-assistance system," in Proc. of IEEE Conference on Intelligent Transportation Systems, 2013, pp. 1993-1999.

[21] J. Jiang and A. Astolfi, "Shared-control for the kinematic model of a rear-wheel drive car," in Proc. of American Control Conference, Chicago, USA, 2015. 